\title{
Protective effects of deep sea water against doxorubicin-induced cardiotoxicity in H9c2 cardiac muscle cells
}

\author{
DO-HYUNG LEE, SOYOUNG KIM and KYUNG-SOO NAM \\ Department of Pharmacology, College of Medicine and Intractable Disease Research Center, \\ Dongguk University, Gyeongju, Gyeongsangbuk-do 780-714, Republic of Korea
}

Received July 14, 2014; Accepted September 1, 2014

DOI: $10.3892 / \mathrm{ijo} .2014 .2666$

\begin{abstract}
Doxorubicin (DOX) is one of the most effective chemotherapeutic agents in the treatment of a variety of tumors. However, its clinical use has been compromised by the risk of cardiotoxicity. Thus, many efforts have been focused on exploring new strategies to prevent or reverse DOX-induced cardiotoxicity. Recently, deep sea water (DSW) has drawn much scientific interest for therapeutic intervention due to its enrichment in nutrients and minerals. In this study, we investigated whether DSW has protective effects against DOX-induced cardiotoxicity. Pre-treatment with DSW significantly increased the viability of DOX-treated rat $\mathrm{H} 9 \mathrm{c} 2$ cardiac muscle cells. This protective effect of DSW appears to be mediated through the inhibition of DNA damage rather than suppression of reactive oxygen species (ROS) production in DOX-treated H9c2 cardiac muscle cells. The inhibitory effect of DSW on DOX-induced DNA damage subsequently attenuated apoptotic signaling such as activation of cysteine-aspartic acid protease-3 (caspase-3) and fragmentation of poly(ADP-ribose) polymerase (PARP), whereas the expression of anti-apoptotic protein B-cell lymphoma-extra large (Bcl-xL) was increased. Moreover, DSW treatment rescued the activation of protein kinase B (Akt) to protect cells from DOX-triggered apoptosis.
\end{abstract}

Correspondence to: Dr Kyung-Soo Nam, Department of Pharmacology, College of Medicine and Intractable Disease Research Center, Dongguk University, 123 Dongdae-ro, Gyeongju, Gyeongsangbuk-do 780-714, Republic of Korea

E-mail: namks@dongguk.ac.kr

Abbreviations: DOX, doxorubicin; DSW, deep sea water; ROS, reactive oxygen species; DCF-DA, 2',7'-dichlorofluorescin diacetate; MDR1, multi-drug resistance protein 1; H2AX, H2A histone family member X; Bcl-xL, B-cell lymphoma-extra large; caspase-3, cysteine-aspartic acid protease-3; PARP, poly(ADPribose) polymerase; Akt, protein kinase B; ERK1/2, extracellular signal-regulated kinase 1/2; GAPDH, glyceraldehyde-3-phosphate dehydrogenase

Key words: doxorubicin, cardiotoxicity, deep sea water, H2AX, $\mathrm{PI} 3 \mathrm{~K} / \mathrm{Akt}$
Taken together, our data showed that DSW has protective effects against DOX-induced cardiotoxicity, suggesting that DSW has some promise as a novel protective supplement for promoting the successful use of DOX in clinical regimen.

\section{Introduction}

The anthracycline antibiotic doxorubicin (DOX) is one of the most effective chemotherapeutic agents in the treatment of numerous solid tumors and hematological malignancies $(1,2)$. However, its clinical use has been compromised due to its dose-dependent cardiotoxicity, which appears to be progressive and permanent $(1,3,4)$. Since the first heart failure was reported in children treated with DOX in 1967 (2), the anthracyclines have been classified as the most common cardiotoxic chemotherapeutic agents. Total dose of DOX within a patient's lifetime is restricted not to exceed $450-500 \mathrm{mg} / \mathrm{m}^{2}$ because cardiotoxicity is related to the cumulative dose (5). Thus, patients who have already received the maximum cumulative dose of DOX cannot receive further DOX therapy even if they may benefit from DOX treatment.

The precise molecular mechanisms underlying anthracycline-induced cardiotoxicity are not fully understood because the cause of cardiotoxicity is complex and multifactorial. The most common hypothesis is that the formation of reactive oxygen species (ROS) such as superoxide anion $\left(\cdot \mathrm{O}_{2}{ }^{-}\right)$ and hydrogen peroxide $\left(\mathrm{H}_{2} \mathrm{O}_{2}\right)$ cause oxidative damage to the cellular components and membranes in heart tissue and reduction of energy in cardiomyocytes, which ultimately lead to cardiomyopathy and congestive heart failure (6-8). In addition to the formation of ROS, more recent studies suggest that cardiotoxicity is associated with DNA damage via DOX interaction with nuclear DNA of cardiac cells, which interferes with DNA replication and transcription, potentially triggering myocyte apoptosis (9-11).

Since DOX-induced cardiotoxicity is a major limiting factor in the use of DOX, new strategies to prevent or reverse the cardiotoxic side-effects of DOX have been explored (12-14). Several antioxidants or iron chelators including N-acetylcysteine, ascorbic acid, and dexrazoxane have been shown to alleviate anthracycline-induced cardiotoxicity by reducing the oxidative stress (15-17). Besides antioxidants, lipid-lowering 
reagents such as probucol and statins also exhibit favorable cardioprotective effects against anthracycline-induced cardiotoxicity (18-20).

Recently, deep sea water (DSW) has gained much scientific interest for therapeutic intervention due to its enrichment in nutrients and minerals. DSW is obtained from a clean area at a depth of $>200 \mathrm{~m}$ and is rich in minerals such as calcium $(\mathrm{Ca})$, magnesium $(\mathrm{Mg})$, potassium $(\mathrm{K})$, sodium $(\mathrm{Na})$, zinc (Zn), etc. (21). Now, it is well recognized that DSW has health benefits in lowering of blood cholesterol and preventing obesity and atherosclerosis $(22,23)$. Moreover, our previous studies showed the inhibitory effects of DSW on the metastatic potential of human breast cancer cell lines (24) and on the carcinogen-induced expression of cyclooxygenase-2 (COX-2), transforming growth factor- $\beta$ (TGF- $\beta$ ), and urokinase plasminogen activator (UPA) in HT-29 colorectal cancer cells (25). Although the precise mechanisms mediating these biological activities of DSW have not been clarified yet, it is presumed that its activities may be derived from the combined ionic action of several minerals. In particular, $\mathrm{Mg}$ and $\mathrm{Ca}$ may play important roles in mediating the biological activities of DSW because they are the main mineral ions present in DSW. Since it is well known that Mg plays important roles in regulating cardiac muscle function and maintaining adequate electrophysiology $(26,27)$, the present study was directed to evaluate the protective effect of DSW against DOX-induced cardiotoxicity in $\mathrm{H} 9 \mathrm{c} 2$ cardiomyocytes.

\section{Materials and methods}

Preparation of DSW. DSW was supplied by the Marine Deep Ocean Water Application Research Center in the Korea Institute of Ocean Science and Technology (Goseong, Gyeongsangnam-do, Korea). DSW was taken from the sea in Goseong at a depth of $500 \mathrm{~m}$ and subjected to a process of filtrations, reverse osmosis, and concentration by electrolysis to achieve desalinated water and 4,000 hardness DSW. Mg and Ca within DSW were present in the ratio of 3:1 and the hardness of DSW was determined from the concentration of $\mathrm{Ca}$ and $\mathrm{Mg}$ ions. The following equation was used to calculate the hardness of DSW in this study: Hardness of DSW $(\mathrm{mg} / \mathrm{l})=\mathrm{M}$ $\mathrm{g}(\mathrm{mg} / \mathrm{l}) \times 4.1+\mathrm{Ca}(\mathrm{mg} / \mathrm{l}) \times 2.5$. DSW of hardness 1,500 was prepared by diluting hardness $4,000 \mathrm{DSW}$ with desalinated DSW (hardness 0) and dissolved Dulbecco's modified Eagle's medium (DMEM) powder with $1 \%$ antibiotic-antimycotic solution. Further serial dilutions were performed to achieve hardness 200-800 DSW media from 1,500 hardness DSW with desalinated media (hardness 0).

Cell culture. $\mathrm{H} 9 \mathrm{c} 2$ rat cardiomyocytes were purchased from the Korean Cell Line Bank (Seoul, Korea). Cells were cultured in DMEM (WelGENE, Daegu, Korea) supplemented with $10 \%$ fetal bovine serum (Invitrogen Life Technologies, Carlsbad, CA, USA) and 1\% antibiotic-antimycotic solution (WelGENE). MCF-7 and MDA-MB-231 human breast cancer cell lines were purchased from the Korean Cell Line Bank. MCF-7 cells were cultured in DMEM supplemented with $10 \%$ fetal bovine serum and $10 \mu \mathrm{g} / \mathrm{ml}$ insulin, while MDA-MB-231 cells were cultured in DMEM supplemented with $10 \%$ fetal bovine serum without insulin.
Cell treatment. After $\mathrm{H} 9 \mathrm{c} 2$ cells were pre-treated with DSW of various hardness for $24 \mathrm{~h}, 0.25 \mu \mathrm{M}$ DOX (Sigma, St. Louis, MO, USA) was added to the cells. Cells were further incubated for $24 \mathrm{~h}$ and harvested for RNA isolation or preparation of protein lysates. For inhibition of the PI3K/Akt-signaling pathway, cells were treated with $10 \mu \mathrm{M}$ LY294002 (LC Laboratories, Woburn, MA, USA) with $0.25 \mu \mathrm{M}$ DOX and cultured for $24 \mathrm{~h}$ before cell harvest.

Cell viability assay. Cells were seeded in 96-well plates and incubated at $37^{\circ} \mathrm{C}$ for $24 \mathrm{~h}$. The cells were treated with conditioned media containing DSW of various hardness $(200,400$, $800,1,500)$ for $24 \mathrm{~h}$ prior to adding $0.25 \mu \mathrm{M}$ DOX. Cells were further incubated for 24 or $48 \mathrm{~h}$ and their cell viabilities were measured by MTT assay (Sigma). Absorbance at $570 \mathrm{~nm}$ was measured in a Multi-Detection Microplate Reader (Molecular Devices, Sunnyvale, CA, USA).

Measurement of intracellular ROS production. After cells were treated as indicated above, cells were trypsinized and incubated with $20 \mu \mathrm{M} 2^{\prime}, 7^{\prime}$-dichlorofluorescin diacetate (DCF-DA) (Sigma) for $1 \mathrm{~h}$ at $37^{\circ} \mathrm{C}$ in the dark. After incubation, cells were immediately washed and resuspended in PBS. Intracellular ROS production was detected on a FACSCalibur II (BD Biosciences, San Jose, CA, USA) by the fluorescent intensity of DCF measured at $525 \mathrm{~nm}$.

Quantitative real-time PCR. The mRNA expression of multi-drug resistance protein 1 (MDR1) was determined by quantitative real-time PCR. Cells were grown and treated in 6-well plates as indicated above. Total RNA was extracted with easy-BLUE $^{\mathrm{TM}}$ Total RNA Extraction kit (Intron Biotechnology, Inc., Gyeonggi, Korea) and cDNA was synthesized with reverse transcriptase (Takara Bio, Inc., Shiga, Japan). The real-time PCR reactions were performed using QuantiMix SYBR-Green kit (Philekorea, Daejeon, Korea) in Eco Real-Time PCR (Illumina, San Diego, CA, USA). mRNA expression level of MDR1 was calculated after normalizing with glyceraldehyde-3-phosphate dehydrogenase (GAPDH). The utilized primer sequences were as follows: MDR1 forward, 5'-GATGGA ATTGATAATGTGGACA-3' and MDR1 reverse, 5'-GTACGTC GTCATCCAGAGC-3'; GAPDH forward, 5'-AACTTTGGCAT CGTGGAAGG-3' and GAPDH reverse, 5'-TACATTGGGGGT AGGAACAC-3'.

Western blot analysis. Cells were grown and treated in 6-well plates as indicated above. Cells were lysed with RIPA buffer (50 mM NaCl, 1\% Triton X-100, 1\% Na deoxycholate, $0.1 \%$ SDS, $50 \mathrm{mM}$ Tris- $\mathrm{HCl} \mathrm{pH} 7.5$ and $2 \mathrm{mM}$ EDTA). Phosphatase and protease inhibitor cocktail (GenDEPOT, Barker, TX, USA) were added immediately before use. Lysates were cleared of debris at $13,000 \mathrm{rpm}$ for $10 \mathrm{~min}$, and protein concentrations were determined using bicinchoninic acid reagent (Sigma). Proteins were separated by SDS-PAGE (8-15\% gels) and transferred onto polyvinylidene difluoride (PVDF) membranes at $100 \mathrm{~V}$ for $45 \mathrm{~min}$. Membranes were blocked in 5\% milk in TBS-Tween $(50 \mathrm{mM}$ Tris- $\mathrm{HCl}$, $150 \mathrm{mM} \mathrm{NaCl}, 0.1 \%$ Tween-20) for $1 \mathrm{~h}$ at room temperature. The following primary antibodies were incubated with blots overnight at $4^{\circ} \mathrm{C}$ : Anti-rabbit phospho- $\mathrm{H} 2 \mathrm{~A}$ histone family 

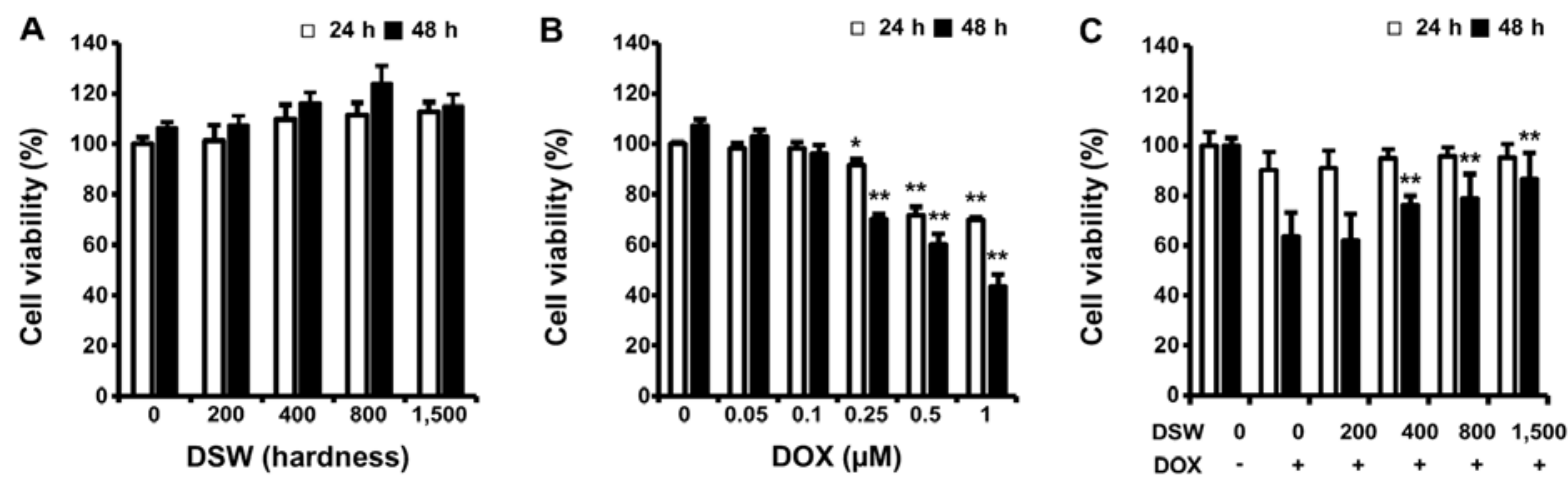

Figure 1. Deep sea water (DSW) protects H9c2 cells from doxorubicin (DOX)-induced cytotoxicity. (A) DSW itself did not induce cytotoxicity when cells were treated with various hardness of DSW for indicated times. (B) DOX-induced cytotoxicity of H9c2 cells exposed to various concentrations of DOX for 24 or $48 \mathrm{~h} .{ }^{*} \mathrm{P}<0.005$ and ${ }^{* *} \mathrm{p}<0.001$ compared to control. (C) DSW at different hardness attenuated DOX-induced cell cytotoxicity when treated with $0.25 \mu \mathrm{M}$ DOX for $48 \mathrm{~h} .{ }^{* *} \mathrm{P}<0.001$ compared to DOX only. The values represent the mean $\pm \mathrm{SD}$.
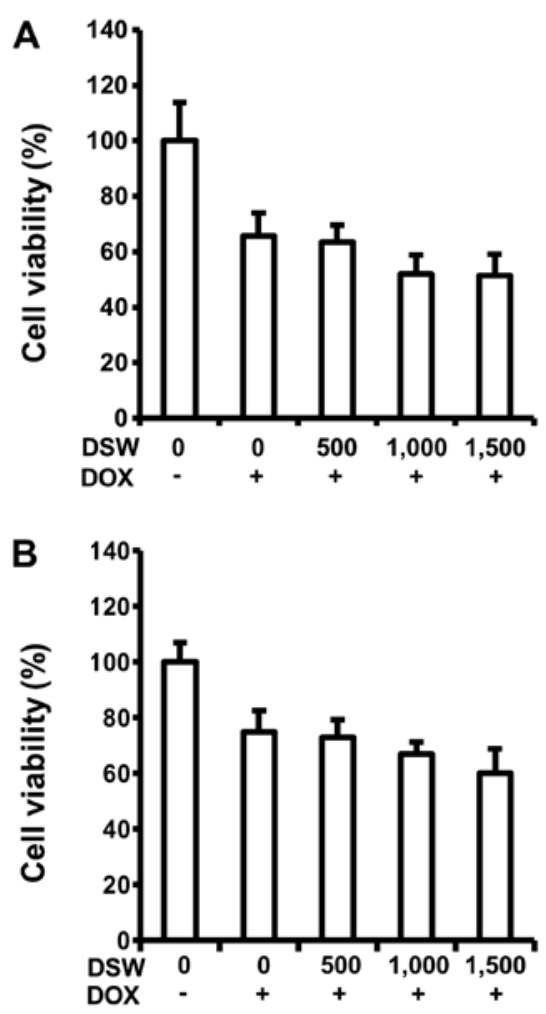

Figure 2. Deep sea water (DSW) enhances antitumor activity of doxorubicin (DOX) in MDA-MB-231 human breast cancer cells. DSW enhanced DOX-induced cell cytotoxicity in (A) MDA-MB-231 and (B) MCF-7 human breast cancer cells when treated with $0.3 \mu \mathrm{M}$ DOX in combination with DSW of different hardness for $72 \mathrm{~h}$. The values represent the mean \pm SD.

member X (H2AX), phospho-p38, total-p38, phospho-protein kinase B (Akt), total-Akt, phospho-extracellular signalregulated kinase $1 / 2($ ERK1/2), total-ERK1/2, B-cell lymphoma-extra large (Bcl-xL), cleaved cysteine-aspartic acid protease-3 (caspase-3), and poly(ADP-ribose) polymerase (PARP) (Cell Signaling Technology, Inc., Beverly, MA, USA). HRP-conjugated secondary anti-rabbit antibody (Santa Cruz Biotechnology, Inc., Santa Cruz, CA, USA) diluted 1:5,000 was incubated with blots for $1 \mathrm{~h}$ at room temperature. Blots were developed using Luminescent Image Analyzer LAS-4000 (Fujifilm, Tokyo, Japan).
Statistical analysis. The Student's t-test was used for statistical analysis of the data. $\mathrm{P}<0.05$ was considered significant.

\section{Results}

DSW protects $H 9 c 2$ rat cardiomyocytes from DOX-induced cell death without interfering with anticancer effects of DOX. To evaluate whether DSW itself has harmful effects on normal cardiomyocyte cells, we first measured viabilities of H9c2 rat cardiomyocytes after treatment with DSW of different hardness for 24 or $48 \mathrm{~h}$. As shown in Fig. 1A, cell proliferation was not affected by treatment with DSW even when cells were treated with DSW of 1,500 hardness for $48 \mathrm{~h}$ (Fig. 1A). However, treatment with DOX significantly reduced the viability of cardiomyocytes dose-dependently (Fig. 1B). To investigate whether DSW is able to protect cardiomyocytes from DOX-induced cell death, we treated cells with conditioned media containing DSW of various hardness for $24 \mathrm{~h}$ prior to adding $0.25 \mu \mathrm{M}$ DOX and further incubated cells for another 24 or $48 \mathrm{~h}$ before measuring cell viability by MTT assay. DSW clearly protected $\mathrm{H} 9 \mathrm{c} 2$ cardiomyocytes from DOX-induced cell death, showing increased cell viability from 60 to $>90 \%$ in the presence of DSW of 1,500 hardness at $48 \mathrm{~h}$ (Fig. 1C).

Since DSW exhibited cardioprotection by inhibiting DOX-induced cell death, we tested possible interference of DSW with antitumor effects of DOX in the MCF-7 and MDA-MB-231 human breast cancer cells. Interestingly, mildly enhanced antitumor effects of DOX were observed in both MCF-7 and MDA-MB-231 cells in a dose-dependent manner, exhibiting $\sim 10 \%$ decrease of cell viability at DSW of 1,500 hardness (Fig. 2A and B). This result manifested that the antitumor effects of DOX were not impaired by DSW. Taken together, our data suggested that DSW provides cardioprotection in cardiomyocytes exposed to DOX, without interfering with the antitumor activity of DOX in human breast cancer cells.

Protective effect of DSW is associated with DNA damage responses rather than ROS generation or expression of MDR1. To elucidate the molecular mechanisms involved in the cardioprotective effects of DSW, we first monitored the generation of ROS in H9c2 cells treated with DOX and DSW 
since a number of recent reviews described the involvement of ROS in the mechanism of DOX-induced cardiotoxicity. In flow cytometry analysis using DCF-DA reagent, which can be converted to fluorescent DCF in a reaction with intracellular ROS, we observed that DOX caused a right-shift of the fluorescence intensity of DCF signal compared to untreated cells, confirming the generation of ROS by DOX. However, this DOX-induced ROS generation was not diminished by pre-treatment with DSW of 1,500 hardness, suggesting that the cardioprotective effects of DSW are independent of ROS generation (Fig. 3A). We also tested whether DSW enhanced the mRNA expression of drug transporter MDR1, which plays an important role in the protection of cardiac tissue by inhibiting accumulation of DOX within tissues (28). Although the expression of MDR1 was induced by stimulation of the cells with DOX, its further induction with DSW treatment was not observed, suggesting that DSW has no effect on inhibiting accumulation of DOX within H9c2 cells (Fig. 3B).

Several studies suggested that induction of DNA damage is an early event in DOX-induced lethal cardiomyocyte injury (10). Since H2AX is rapidly phosphorylated in response to DNA damage and its phosphorylation is frequently used as a marker for DNA damage (29), we analyzed H2AX phosphorylation $(\gamma-\mathrm{H} 2 \mathrm{AX})$ by western blot analysis to test whether the cardioprotective effects of DSW are associated with the response of DNA damage in H9c2 cells. As expected, DOX exposure significantly induced the phosphorylation of H2AX. However, pre-treatment with DSW attenuated this response in a dose-dependent manner, exhibiting $\sim 50 \%$ decreased phosphorylation of H2AX at DSW of 1,500 hardness (Fig. 3C).

The inhibitory effect of DSW on DOX-induced DNA damage subsequently attenuates apoptotic signaling. As DSW suppressed DOX-induced DNA damage, which triggers the cell death program, we assessed the effects of DSW on apoptosis signaling. We first analyzed the expression of Bcl-xL, which is an anti-apoptotic protein that inhibits the release of mitochondrial cytochrome $c$ into the cytosol. Western blot analysis demonstrated that DOX downregulated the expression of Bcl-xL, which was effectively restored to control level by DSW of 1,500 hardness (Fig. 4A). We further studied the inhibitory effect of DSW on DOX-triggered apoptosis by evaluating the activation of caspase-3 and PARP fragmentation. Treatment of cardiomyocytes with DOX resulted in a remarkable increase in the levels of cleaved caspase-3, as well as fragmentation of its substrate, PARP. However, DSW effectively suppressed the activation of caspase-3 (Fig. 4B), leading to subsequent inhibition of PARP fragmentation (Fig. 4C). These data suggest that DSW can counteract DOX-triggered apoptosis in $\mathrm{H} 9 \mathrm{c} 2$ cells.

DSW rescues the Akt-signaling pathway to protect cells from DOX-induced cell death. Several studies have shown that the PI3K/Akt- and MAP kinase-signaling pathways are involved in DOX-induced apoptosis $(11,30,31)$. Increased MEK-ERK1/2 activity is responsible for the DOX-induced apoptosis (11), while increased PI3K/Akt activity is protective against DOX-induced cardiomyocyte apoptosis (31). Thus, to get more detailed insights into the mechanism underlying the protective effects of DSW against DOX-induced apoptosis, we further analyzed the effects of DSW on PI3K/Akt- and
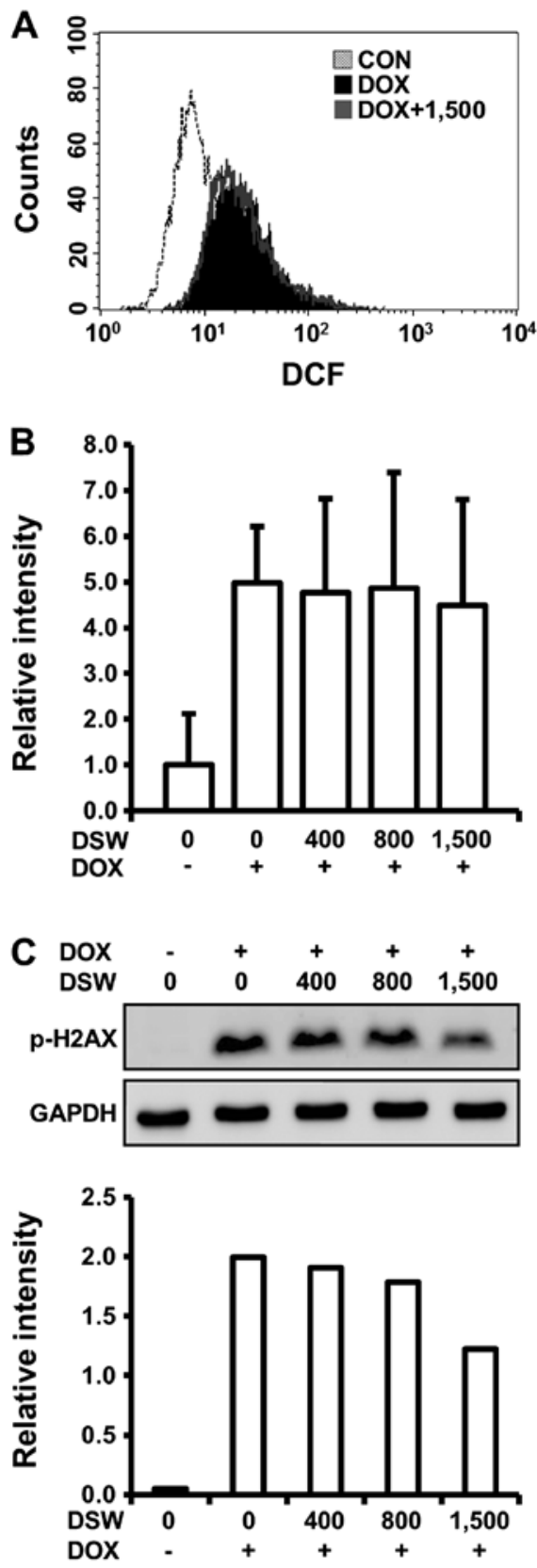

Figure 3. Protective effect of deep sea water (DSW) is associated with DNA damage responses rather than reactive oxygen species (ROS) generation or expression of multi-drug resistance protein 1 (MDR1). (A) The generation of ROS was induced by $24 \mathrm{~h}$ treatment with $0.25 \mu \mathrm{M}$ doxorubicin (DOX) in H9c2 cells pre-treated with DSW of 1,500 hardness. (B) MDR1 gene expression of cells pre-treated with DSW of various hardness for $24 \mathrm{~h}$ and co-incubated with $0.25 \mu \mathrm{M}$ DOX for $24 \mathrm{~h}$. The values represent the mean $\pm \mathrm{SD}$. (C) H2A histone family member X (H2AX) phosphorylation was analyzed by western blot analysis. Glyceraldehyde-3-phosphate dehydrogenase (GAPDH) was used as an internal control and the relative intensity of each sample was expressed as fold change compared to control after normalization to respective internal control.

MAP kinase-signaling pathways. Although DOX significantly induced phosphorylation of ERK1/2, no inhibitory effect of DSW on the protein level of phosphorylated ERK1/2 was observed (Fig. 5A). Similar induction was observed in the phosphorylation of p38 in DOX-treated cardiomyocytes. However, treatment with DSW significantly decreased the phosphorylation of $\mathrm{p} 38$ (Fig. 5B), suggesting that DSW protects 

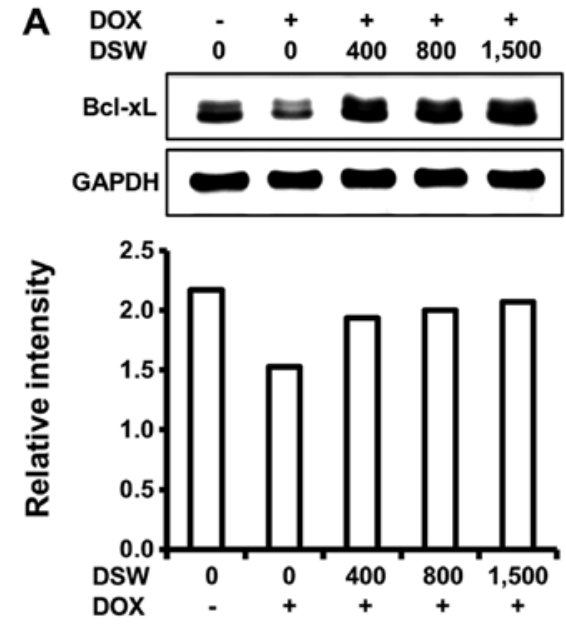

$\begin{array}{llllccc}\text { B } & \text { Dox } & - & + & + & + & + \\ \text { DSW } & 0 & 0 & 400 & 800 & 1,500\end{array}$
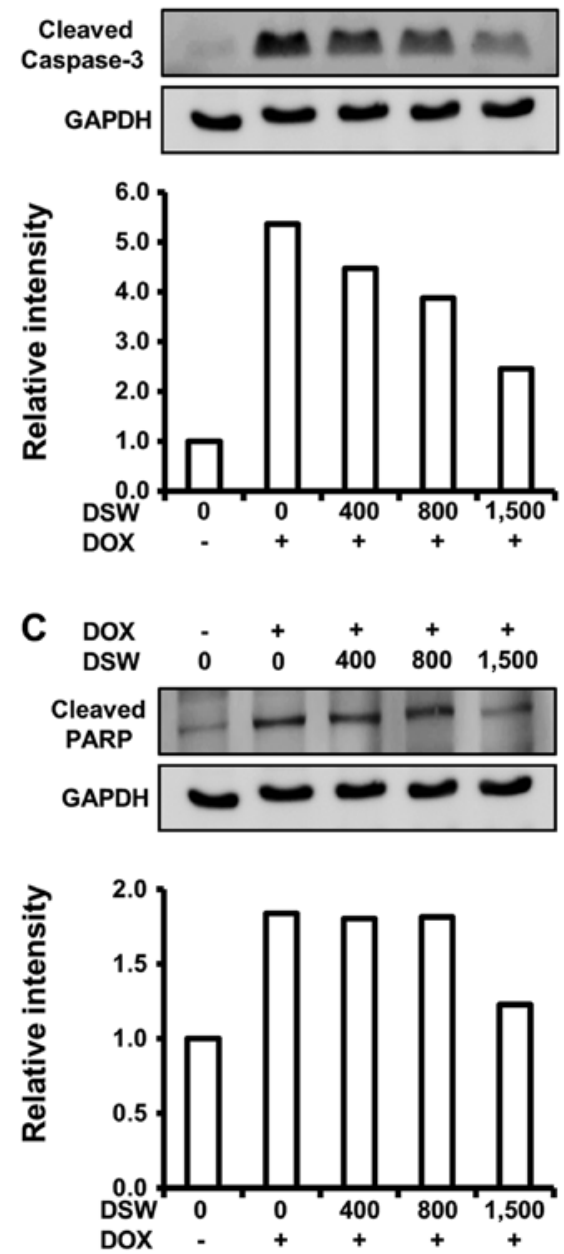

Figure 4. Deep sea water (DSW) inhibits doxorubicin (DOX)-induced apoptosis signal. Protein lysates were prepared from cells treated with DSW of different hardness in the presence of $0.25 \mu \mathrm{M}$ DOX. The expression of (A) B-cell lymphoma-extra large (Bcl-xL), (B) cleaved cysteine-aspartic acid protease-3 (caspase-3), and (C) fragmented poly(ADP-ribose) polymerase (PARP) was analyzed by western blot analysis. Glyceraldehyde-3-phosphate dehydrogenase (GAPDH) was used as an internal control and the relative intensity of each sample was expressed as fold change compared to control after normalization to respective internal control.

cells from DOX-induced cell death by suppressing the activation of $\mathrm{p} 38$. In contrast to the roles of MAP kinase-signaling
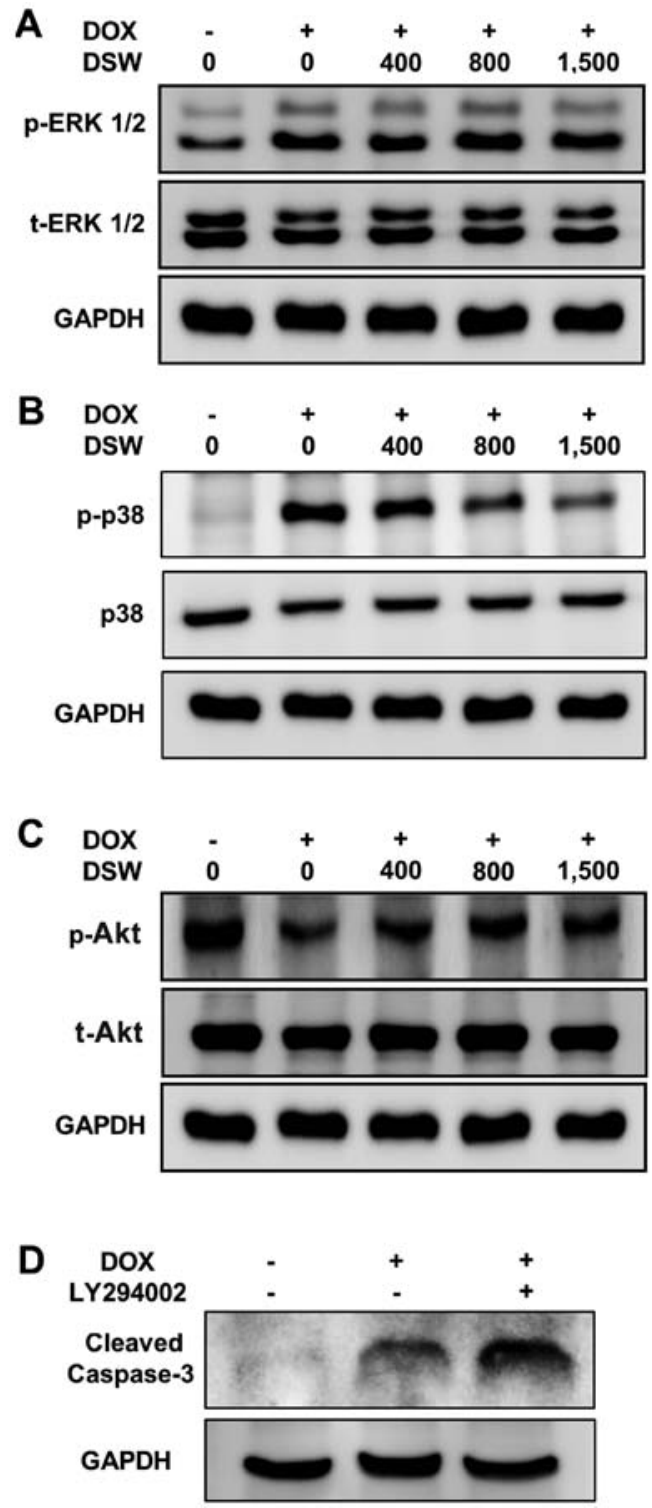

Figure 5. Deep sea water (DSW) rescues the protein kinase B (Akt)-signaling pathway to protect cells from doxorubicin (DOX)-induced cell death. Cells were treated with conditioned media containing DSW of different hardness for $24 \mathrm{~h}$ prior to addition of $0.25 \mu \mathrm{M}$ DOX. Cells were further incubated for $24 \mathrm{~h}$ and harvested for protein lysates. (A) DSW did not alter the expression of phosphorylated extracellular signal-regulated kinase 1/2 (ERK1/2). (B) Treatment with DSW significantly decreased the phosphorylation of p38. (C) DSW treatment rescued the activation of Akt from DOX-mediated Akt suppression. (D) Blocking the PI3K/Akt-signaling pathway with LY294002 significantly increased the cleavage of cysteine-aspartic acid protease-3 (caspase-3). Glyceraldehyde-3-phosphate dehydrogenase (GAPDH) was used as an internal control.

pathways, the PI3K/Akt-signaling pathway is a major cell survival signal in cardiomyocytes (31-33). Our data also showed that DOX significantly decreased the phosphorylation of Akt. However, DSW treatment rescued the activation of Akt from DOX-mediated Akt suppression (Fig. 5C). To confirm the protective role of PI3K/Akt-signaling pathway against DOX-induced apoptosis, we blocked this signal with LY294002. Blocking PI3K/Akt-signaling pathway with LY294002 in DOX-treated H9c2 cells significantly increased the cleavage of caspase-3 (Fig. 5D), resulting in decreased cell viability (data not shown). These data suggest that the PI3K/Akt-signaling 
pathway is protective against the progression of apoptosis and DSW rescues cardiomyocytes from DOX-induced cell death via the restoration of Akt activation.

\section{Discussion}

In the present study, we demonstrated that DSW provides a cardioprotective effect against DOX-induced cardiotoxicity in rat $\mathrm{H} 9 \mathrm{c} 2$ cardiac muscle cells without interfering with the antitumor activities of DOX. This protective effect of DSW appears to be mediated through the inhibition of DNA damage rather than suppression of ROS, resulting in subsequent inhibition of DOX-induced apoptotic signaling. Moreover, DSW rescues the Akt-signaling pathway to protect cells from DOX-induced cell death.

Since oxidative stress is generally accepted as the major mechanism by which DOX causes toxicity to the heart, numerous antioxidants have been investigated as cardioprotective agents to prevent or reverse the cardiotoxic side-effects of DOX. However, administration of antioxidants with DOX has failed to show favorable outcomes in clinical studies, implying the involvement of additional mechanisms in the cardiotoxic action of DOX. More recent studies suggest that DNA damage plays an important role in mediating DOX-induced cardiomyocyte death through a pathway involving p53 and the mitochondria (10). A cardioprotective effect through direct inhibition of DOX-induced DNA damage was demonstrated by Huelsenbeck et al showing that lovastatin, which is a widely used lipid-lowering drug, effectively protects against DOX-induced cardiac damage by inhibiting DNA damage in a Rac1-dependent manner without suppressing ROS generation in both in vitro and in vivo studies (18). Since DSW also exhibits a cardioprotective effect through the inhibition of DNA damage rather than ROS generation, inhibiting DOX-induced DNA damage may provide alternative cardioprotective strategies besides antioxidant therapy in DOX treatment.

It is not clear which component of DSW is responsible for the protective effects against DOX-induced cardiotoxicity. However, it is assumed that the combined ionic action of several minerals such as $\mathrm{Ca}, \mathrm{Mg}, \mathrm{K}$, and $\mathrm{Na}$ may play important roles in mediating diverse biological effects of DSW including its cardioprotective effect. Indeed, it is now well known that these essential metal ions are crucial to maintain cellular functions and their deficiency is considered to be a potential health hazard. In particular, $\mathrm{Mg}$ and $\mathrm{Ca}$ may be the primary minerals responsible for the protective effect of DSW against DOX-induced cardiotoxicity due to their profound existence within DSW. Mg is an essential intracellular ion necessary for normal cellular function (26). Its crucial role in regulating cardiac function is well established in studies of several cardiovascular diseases, including hypertension, stroke and atherosclerosis $(34,35)$. Moreover, it has been shown that chronic dietary $\mathrm{Mg}$ deficiency results in cardiac apoptosis in the rat heart (27), whereas $\mathrm{Mg}$ treatment contributes to the improvement of the prognosis of heart failure patients (36). Ca deficiency is also known to be associated with increased apoptosis in multiple cell lines. Although it has been suggested that an excessive level of intracellular ionized Ca may be responsible for triggering apoptotic machinery within cells, emerging evidence emphasizes that loss of $\mathrm{Ca}$ is a greater determinant in apoptotic cell death than high level of intracellular ionized $\mathrm{Ca}(37,38)$. The requirement of both $\mathrm{Mg}$ and $\mathrm{Ca}$ in preventing apoptotic cell death has been proven by Feng et al (39). They demonstrated that deficiency of $\mathrm{Mg}$ and $\mathrm{Ca}$ induce apoptosis in Chinese hamster ovary cells but the restoration of $\mathrm{Mg}$ and Ca protects cells from apoptotic cell death. Furthermore, a clinical study has shown that supplementation of $\mathrm{Mg}$ and $\mathrm{Ca}$ in drinking water decreased the mortality of breast cancer patients (40). In this study, the cardioprotective effect of DSW was escalated corresponding to the hardness of DSW, which reflects the concentration of $\mathrm{Ca}$ and $\mathrm{Mg}$. Compared to the effect of desalinated DSW (hardness 0), the cardioprotective effect was maximized in cardiomyocytes treated with 1,500 hardness DSW, in which Ca concentration is $\sim 100 \mathrm{mg} / \mathrm{l}$ and the amount of $\mathrm{Mg}$ is $\sim 300 \mathrm{mg} / \mathrm{l}$. Thus, the synergistic action of $\mathrm{Mg}$ and Ca within DSW may be mainly responsible for its cardioprotective effects against DOX-induced cell death. However, the possible involvement of the other trace elements in DSW also needs to be considered. Our data showed that inhibition of DOX-induced DNA damage is one of the mechanisms underlying the cardioprotective effects of DSW. To elucidate a more detailed mechanism of DSW-mediated cardioprotection against DOX-induced cardiotoxicity, further investigations will be needed. So far, diverse beneficial effects of DSW have been revealed and applied in many fields such as food processing and cosmetics (21). However, to our knowledge, this is the first study to show the cardioprotective effects of DSW against DOX-induced cardiotoxicity, suggesting that DSW has some promise as a novel protective supplement to extend the use of DOX therapy in patients who may benefit from further DOX treatment.

\section{Acknowledgements}

This study was supported by the project entitled 'Development of Technology for support of DSW industry (PJT200014)' from the Ministry of Land, Transport and Maritime Affairs, Korea.

\section{References}

1. Minotti G, Menna P, Salvatorelli E, Cairo G and Gianni L: Anthracyclines: molecular advances and pharmacologic developments in antitumor activity and cardiotoxicity. Pharmacol Rev 56: 185-229, 2004.

2. Tan C, Tasaka H, Yu KP, Murphy ML and Karnofsky DA: Daunomycin, an antitumor antibiotic, in the treatment of neoplastic disease. Clinical evaluation with special reference to childhood leukemia. Cancer 20: 333-353, 1967.

3. Gharib MI and Burnett AK: Chemotherapy-induced cardiotoxicity: current practice and prospects of prophylaxis. Eur J Heart Fail 4: 235-242, 2002.

4. Singal PK, Li T, Kumar D, Danelisen I and Iliskovic N: Adriamycin-induced heart failure: mechanism and modulation. Mol Cell Biochem 207: 77-86, 2000.

5. Von Hoff DD, Layard MW, Basa P, et al: Risk factors for doxorubicin-induced congestive heart failure. Ann Intern Med 91: 710-717, 1979.

6. Rajagopalan S, Politi PM, Sinha BK and Myers CE: Adriamycin-induced free radical formation in the perfused rat heart: implications for cardiotoxicity. Cancer Res 48: 4766-4769, 1988. 
7. Simůnek T, Stérba M, Popelová O, Adamcová M, Hrdina R and Gersl V: Anthracycline-induced cardiotoxicity: overview of studies examining the roles of oxidative stress and free cellular iron. Pharmacol Rep 61: 154-171, 2009.

8. Vásquez-Vivar J, Martasek P, Hogg N, Masters BS, Pritchard KA Jr and Kalyanaraman B: Endothelial nitric oxide synthase-dependent superoxide generation from adriamycin. Biochemistry 36 : 11293-11297, 1997.

9. Goto S, Ihara Y, Urata Y, et al: Doxorubicin-induced DNA intercalation and scavenging by nuclear glutathione S-transferase pi. FASEB J 15: 2702-2714, 2001.

10. L'Ecuyer T, Sanjeev S, Thomas R, et al: DNA damage is an early event in doxorubicin-induced cardiac myocyte death. Am J Physiol Heart Circ Physiol 291: H1273-H1280, 2006.

11. Liu J, Mao W, Ding B and Liang CS: ERKs/p53 signal transduction pathway is involved in doxorubicin-induced apoptosis in H9c2 cells and cardiomyocytes. Am J Physiol Heart Circ Physiol 295: H1956-H1965, 2008.

12. Chularojmontri L, Gerdprasert O and Wattanapitayakul SK: Pummelo protects doxorubicin-induced cardiac cell death by reducing oxidative stress, modifying glutathione transferase expression, and preventing cellular senescence. Evid Based Complement Alternat Med 2013: 254835, 2013.

13. Das J, Ghosh J, Manna P and Sil PC: Taurine suppresses doxorubicin-triggered oxidative stress and cardiac apoptosis in rat via up-regulation of PI3-K/Akt and inhibition of p53, p38-JNK. Biochem Pharmacol 81: 891-909, 2011.

14. Ghosh J, Das J, Manna P and Sil PC: The protective role of arjunolic acid against doxorubicin induced intracellular ROS dependent JNK-p38 and p53-mediated cardiac apoptosis. Biomaterials 32: 4857-4866, 2011.

15. Doroshow JH, Locker GY, Ifrim I and Myers CE: Prevention of doxorubicin cardiac toxicity in the mouse by $\mathrm{N}$-acetylcysteine. J Clin Invest 68: 1053-1064, 1981.

16. Shimpo K, Nagatsu T, Yamada K, et al: Ascorbic acid and adriamycin toxicity. Am J Clin Nutr 54 (Suppl 6): 1298S-1301S, 1991

17. Swain SM and Vici P: The current and future role of dexrazoxane as a cardioprotectant in anthracycline treatment: expert panel review. J Cancer Res Clin Oncol 130: 1-7, 2004.

18. Huelsenbeck J, Henninger C, Schad A, Lackner KJ, Kaina B and Fritz G: Inhibition of Racl signaling by lovastatin protects against anthracycline-induced cardiac toxicity. Cell Death Dis 2: e190, 2011.

19. Siveski-Iliskovic N, Hill M, Chow DA and Singal PK: Probucol protects against adriamycin cardiomyopathy without interfering with its antitumor effect. Circulation 91: 10-15, 1995.

20. Siveski-Iliskovic N, Kaul N and Singal PK: Probucol promotes endogenous antioxidants and provides protection against adriamycin-induced cardiomyopathy in rats. Circulation 89 2829-2835, 1994.

21. Nakasone T and Akeda S: The application of deep sea water in Japan. UJNR Technical Report 28: 69-75, 1999.

22. Hwang HS, Kim SH, Yoo YG, et al: Inhibitory effect of deep-sea water on differentiation of 3T3-L1 adipocytes. Mar Biotechnol (NY) 11: 161-168, 2009.

23. Radhakrishnan G, Yamamoto M, Maeda $\mathrm{H}$, et al: Intake of dissolved organic matter from deep sea water inhibits atherosclerosis progression. Biochem Biophys Res Commun 387: $25-30,2009$.
24. Kim S, Chun SY, Lee DH, Lee KS and Nam KS: Mineral-enriched deep-sea water inhibits the metastatic potential of human breast cancer cell lines. Int J Oncol 43: 1691-1700, 2013.

25. Lee KS, Shin JS, Kwon YS, Moon DS and Nam KS: Suppression of cancer progression and metastasis in HT-29 human colorectal adenocarcinoma by deep sea water. Biotechnol Bioproc Eng 18 194-200, 2013

26. Hartwig A: Role of magnesium in genomic stability. Mutat Res 475: 113-121, 2001.

27. Tejero-Taldo MI, Chmielinska JJ and Weglicki WB: Chronic dietary $\mathrm{Mg}^{2+}$ deficiency induces cardiac apoptosis in the rat heart. Magnes Res 20: 208-212, 2007.

28. van Asperen J, van Tellingen O, Tijssen F, Schinkel AH and Beijnen JH: Increased accumulation of doxorubicin and doxorubicinol in cardiac tissue of mice lacking mdrla P-glycoprotein. Br J Cancer 79: 108-113, 1999.

29. Olive PL: Detection of DNA damage in individual cells by analysis of histone H2AX phosphorylation. Methods Cell Biol 75: 355-373, 2004.

30. Brantley-Finley C, Lyle CS, Du L, et al: The JNK, ERK and p53 pathways play distinct roles in apoptosis mediated by the antitumor agents vinblastine, doxorubicin, and etoposide. Biochem Pharmacol 66: 459-469, 2003.

31. Negoro S, Oh H, Tone E, et al: Glycoprotein 130 regulates cardiac myocyte survival in doxorubicin-induced apoptosis through phosphatidylinositol 3-kinase/Akt phosphorylation and Bcl-xL/caspase-3 interaction. Circulation 103: 555-561, 2001.

32. Ahmed NN, Grimes HL, Bellacosa A, Chan TO and Tsichlis PN: Transduction of interleukin-2 antiapoptotic and proliferative signals via Akt protein kinase. Proc Natl Acad Sci USA 94: 3627-3632, 1997.

33. Wang Y: Mitogen-activated protein kinases in heart development and diseases. Circulation 116: 1413-1423, 2007.

34. Saris NE, Mervaala E, Karppanen H, Khawaja JA and Lewenstam A: Magnesium. An update on physiological, clinical and analytical aspects. Clin Chim Acta 294: 1-26, 2000.

35. Song Y, Manson JE, Cook NR, Albert CM, Buring JE and Liu S: Dietary magnesium intake and risk of cardiovascular disease among women. Am J Cardiol 96: 1135-1141, 2005.

36. Almoznino-Sarafian D, Sarafian G, Berman S, et al: Magnesium administration may improve heart rate variability in patients with heart failure. Nutr Metab Cardiovasc Dis 19: 641-645, 2009

37. Kluck RM, McDougall CA, Harmon BV and Halliday JW: Calcium chelators induce apoptosis - evidence that raised intracellular ionised calcium is not essential for apoptosis. Biochim Biophys Acta 1223: 247-254, 1994.

38. Turner CP, Connell J, Blackstone K and Ringler SL: Loss of calcium and increased apoptosis within the same neuron. Brain Res 1128: 50-60, 2007.

39. Feng H, Guo L, Gao H and Li XA: Deficiency of calcium and magnesium induces apoptosis via scavenger receptor BI. Life Sci 88: 606-612, 2011.

40. Yang CY, Chiu HF, Cheng MF, Hsu TY and Wu TN: Calcium and magnesium in drinking water and the risk of death from breast cancer. J Toxicol Environ Health A 60: 231-241, 2000. 